\title{
IDENTIFICACIÓN DE PRESENCIA DE CEMENTO ALUMINOSO EN HORMIGONES MEDIANTE EL USO DEL PACHÓMETRO
}

\author{
(IDENTIFICATION OF HIGH ALUMINA CEMENT IN CONCRETE BY THE USE \\ OF THE PACHOMETER)
}

C. Andrade y J. L. Lozano

Instituto de Ciencias de la Construcción "Eduardo Torroja" del CSiC - Madrid.

\section{Seguí y E. Vicens}

Escuela Técnica Superior de Arquitectura del Vallés - Barcelona

\section{E. Hernández}

ITEC - Barcelona
ESPAÑA

\section{RESUMEN}

La constatación de los daños que se producen en estructuras de hormigón de cemento aluminoso, debido a su rápida transformación y carbonatación, ha llevado a intentar poner a punto diversos métodos de detección acelerada in situ de la presencia del citado cemento. En el presente trabajo se aportan unos primeros resultados sobre el empleo de métodos magnéticos (pachómetro) habituales en la medida del espesor del recubrimiento de armaduras para identificar si un hormigón está fabricado o no con cemento aluminoso. Los resultados de laboratorio se han contrastado en obra pudiéndose comprobar que el pachómetro utilizado es capaz de detectar perfectamente la presencia de aluminoso previa calibración con un hormigón de portland puro. Esta investigación preliminar también ha permitido comprobar que los hormigones de cenizas volantes presentan, como el aluminoso, una menor reluctancia magnética.

\section{SUMMARY}

The observation of the premature degradations detected in High Alumina concretes due to the transformation and carbonation of this kind of cement has promoted the interest to develop in-situ testing methods for the fast identification of HAC presence. In present work, preliminary results are given on the use of magnetic methods (pachometer), usually employed in the concrete cover verification, to identify the existence of HAC in a concrete. The laboratory results have been verified in practice and show that HAC can be clearly distinguished of an OPC concrete with the pachometer used in present experimentation. The research has also indicated that fly ash cements as aluminous one, have a lower magnetic reluctance.

\section{INTRODUCCIÓN}

Los recientes casos de patologías aparecidos fundamentalmente en Cataluña debidos en principio al uso inapropiado de cemento aluminoso en viguetas de forjados, ha generado la necesidad de identificar de forma rápida y barata la presencia de cemento aluminoso en hormigones endurecidos.

Si bien es importante resaltar que las patologías descubiertas (pérdidas de resistencias mecánicas, carbonatación del hormigón y corrosión de las armaduras (1) ) no son exclusivas del cemento aluminoso, sino que aparecen también en hormigones de mala calidad fa. bricados con cemento tipo portland, si es necesario reconocer que el riesgo de que aparezcan desórdenes es, en teoría, mayor en el caso del aluminoso. Asi pues, una identificación rápida en obra puede contribuir a un dictamen general sobre la vida residual o la necesidad de mantenimiento futuro de un edificio.

Varios son los métodos posibles que existen para identificar con seguridad la presencia de cemento aluminoso en un hormigón: análisis químico tradicional, análisis por Rayos X, por Análisis Térmico Diferencial, por Espectropía de Infrarrojos, etc (2). En general todos ellos implican la necesidad de acudir a laboratorios especializados con equipos costosos y sofisticados. 


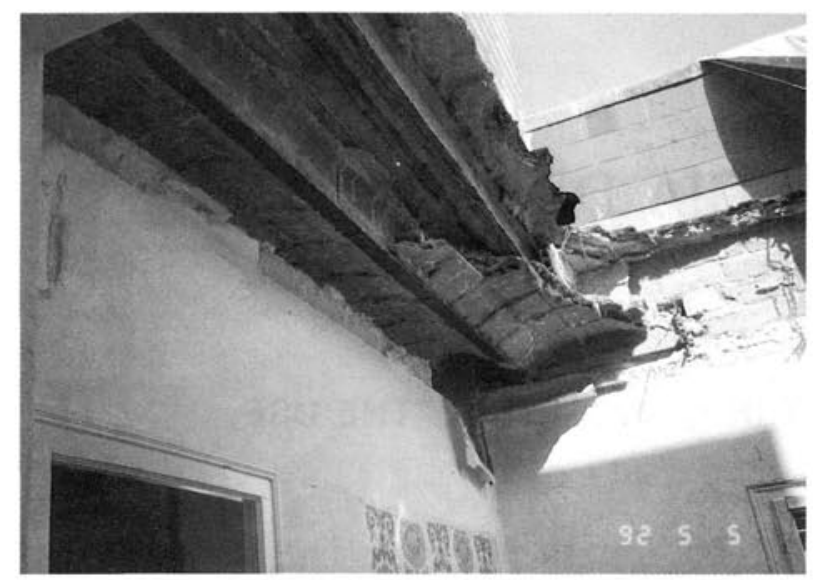

Cubierta que colapsó en el Turó de la Peira de Barcelona.

A pie de obra se está utilizando en la actualidad la simple observación visual del color y el llamado método de la oxina (3). Ambos métodos son susceptibles de inducir a error. El color, porque tanto los áridos finos empleados, como el tipo de encofrado que se usó pueden hacer variar la gama de colores habituales y el color del hormigón en su superficie puede resultar completamente diferente al del interior. También porque en ocasiones se empleó mezcla de cemento aluminoso con portland, lo que se hace muy difícil de detectar por el color.

En cuanto a la oxina, parece que la posible carbonatación introduce un factor de error que puede invalidar la prueba.

Así pues, sigue siendo de interés la búsqueda de métodos rápidos y económicos que pudieran ser aplicados a pie de obra. En esta búsqueda los autores del presente trabajo radicados en Barcelona, encontraron que los hormigones de cemento aluminoso eran sensibles a la prueba del pachómetro. Es decir, se magnetizaban y hacian mover la aguja de estos aparatos, aun en ausencia total de armaduras cercanas.

Esta evidencia experimental llevó a diseñar una pequeña investigación para conocer la viabilidad de utilización de esta técnica, de la que se va a dar cuenta a continuación.

\section{Principio de funcionamiento del pachómetro}

La interacción entre los campos electromagnéticos y los conductores constituye el principio en el que se basan estos aparatos y que permiten la detección de forma no destructiva de la presencia de armaduras así como su dirección y, en ocasiones, la magnitud del espesor de recubrimiento.

El principio es conocido desde antiguo y se basa en la aplicación a la superficie del hormigón de un detector o palpador constituido por una bobina recorrida por ( ) Consejo Superior de Investigaciones Científicas una corriente alterna que genera un campo electromagnético. Cuando existe un conductor eléctrico en sus proximidades, se cierra el circuito y el conjunto actúa como un transformador, lo que se refleja en el paso de una corriente. Es decir, se basan en la medición de la distorsión del campo magnético del transductor (palpador) producida por la presencia en el entorno de un material de alta permeabilidad magnética (4) como son el hierro, niquel, etc.

Esta forma de operar aparentemente tan simple se aplica en muchos campos, no sólo en la construcción. Así se emplea para la detección de metales, para la detección de defectos en recubrimientos metálicos o grietas y fisuras. La base de su sensibilidad se halla en la frecuencia utilizada en la corriente alterna.

En el hormigón son ampliamente utilizados como detectores de armaduras y con algunos modelos más sofisticados, como medida no destructiva del espesor de recubrimiento (con más o menos error según los aparatos y hormigones).

Al percibir que el pachómetro usado sobre cemento aluminoso no permitía identificar con claridad la posición de las armaduras y que en ausencia de ellas el hormigón también presentaba propiedades ferromagnéticas, se pensó que el efecto tenía que ser debido a la mucha mayor proporción de óxidos férricos (aluminato-ferritos cálcicos) que contiene el cemento aluminoso, y que de alguna forma estos compuestos presentan baja reluctancia magnética, como es sabido que tienen alguna de las variedades de los óxidos de hierro.

Así, como ya se ha indicado, se diseñó un plan experimental para evaluar en el laboratorio el alcance y carácter sistemático de este comportamiento.

\section{METODOLOGÍA EXPERIMENTAL}

\section{Materiales}

Se fabricaron una serie de probetas de mortero de $4 \times 4 \times 16 \mathrm{~cm}$ con las proporciones de cemento aluminoso y cemento portland mezclados que se indican en el Cuadro 1. Todos los morteros se amasaron con agua filtrada desionizada y con relación $a / c=0,5$ (considerando como cemento la suma total del aluminoso + portland).

\section{Cuadro 1}

\begin{tabular}{|l|r|r|r|r|r|r|}
\hline probeta n. & 1 & 2 & 3 & 4 & 5 & 6 \\
\hline cemento portland \% & 0 & 20 & 40 & 60 & 80 & 100 \\
\hline cemento aluminoso \% & 100 & 80 & 60 & 40 & 20 & 0 \\
\hline
\end{tabular}


También se fabricaron 2 probetas cilindricas de hormigón de $15 \times 30 \mathrm{~cm}$, una con $350 \mathrm{~kg}$ de cemento por $\mathrm{m}^{3}$ de hormigón y la otra con $250 \mathrm{~kg}$, ambas elaboradas únicamente con cemento aluminoso. La dosificación de ambos hormigones se ajustó por el método de Fuller. Ambas probetas fueron vibradas. Las cantidades de los distintos componentes aparece en el Cuadro n. 2.

\section{Cuadro 2}

\begin{tabular}{|l|r|r|}
\hline & \multicolumn{1}{|c|}{ A } & \multicolumn{1}{|c|}{ B } \\
\hline Cemento & $350 \mathrm{~kg} / \mathrm{m}^{3}$ & $250 \mathrm{~kg} / \mathrm{m}^{3}$ \\
Arena $(0-6 \mathrm{~mm})$ & $850 \mathrm{~kg} / \mathrm{m}^{3}$ & $989 \mathrm{~kg} / \mathrm{m}^{3}$ \\
Gravilla $(6-12 \mathrm{~mm})$ & $1.057 \mathrm{~kg} / \mathrm{m}^{3}$ & $1.137 \mathrm{~kg} / \mathrm{m}^{3}$ \\
Agua & $175 \mathrm{I}$. & $125 \mathrm{I}$. \\
\hline
\end{tabular}

Una vez fabricadas todas las probetas se curaron en cámara húmeda durante 24 horas y luego se conservaron en distintas condiciones de humedad como luego se indica en el Cuadro n. 3.

\section{Aparatos empleados}

El aparato empleado aparece en la fig. 1 y se trata de un pachómetro marca KOLECTRY de Londres, que permite una medida cuantitativa muy precisa de la reluctancia al campo magnético generado por el sensor o palpador. En la escala usada permite identificar profundidades de armadura (espesor de recubrimiento) de 0 a $10 \mathrm{~cm}$, de tal forma que, cuanto más profunda se encuentra la armadura, menor es el desplazamiento de la aguja.

Dado que expresar los resultados en la escala de espesores de recubrimiento podia inducir a error (a más contenido en óxidos magnéticos del hormigón, la aguja iba a indicar un número más pequeño de menor espesor de recubrimiento), se decidió utilizar la escala que tiene el aparato para indicar el nivel de carga de las baterías que está graduada de 1 a 100. Así, un mayor contenido en óxidos magnéticos se indicará con un número mayor en una escala de 1 a 100.

Las medidas se efectuaban colocando el palpador directamente en contacto con la superficie sobre la probeta. En el caso de las de mortero la superficie del palpador era menor a la de la probeta sobresaliendo por los laterales de ella, cosa que no ocurría en las de hormigón. En éstas el palpador se colocó sobre la cara superior plana (sobre la generatriz la medida era idéntica).

Para corregir el efecto de que el palpador resultara de superficie diferente a la de la parte lateral de las pro๑) Consejo Superior de Investigaciones Científicas

Licencia Creative Commons 3.0 España (by-nc)

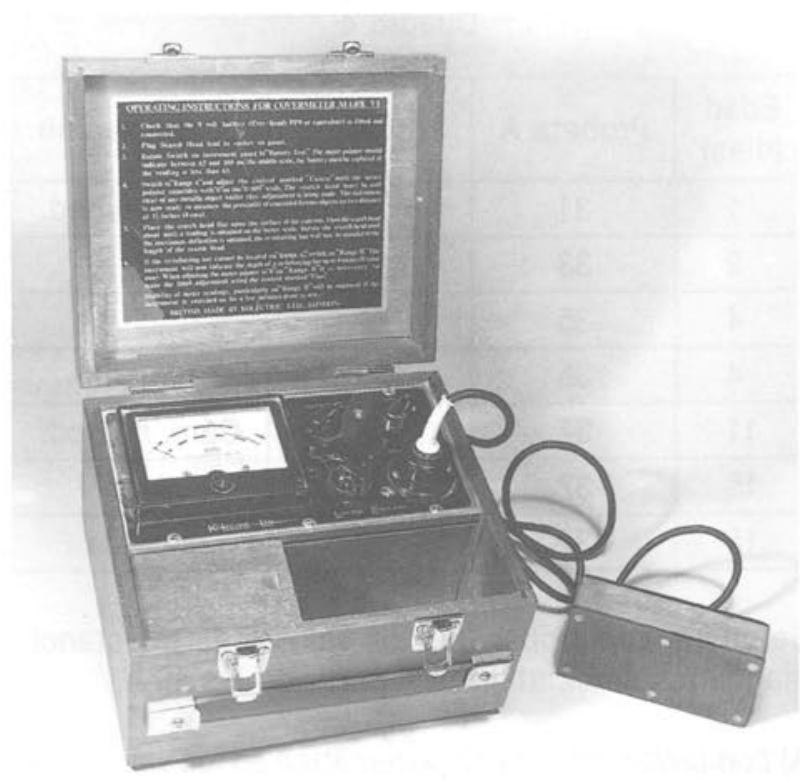

Fig. 1

betas de mortero, se calculó el factor de corrección siguiente:

$\frac{\text { área de una cara lateral de la probeta }}{\text { área de contacto del palpador }}=\frac{64}{48}=1,333$

En todos los casos la primera medida se efectuó una vez desenmoldadas las probetas a las $24 \mathrm{~h}$.

\section{RESULTADOS}

Los resultados obtenidos en el caso de las probetas de mortero aparecen en el Cuadro n. 3 y en el 4 las correspondientes a las de hormigón.

\section{Cuadro 3}

\begin{tabular}{|c|c|c|c|c|c|c|c|}
\hline \multirow[b]{2}{*}{$\begin{array}{l}\text { Edad } \\
\text { (días) }\end{array}$} & \multicolumn{6}{|c|}{ n. probeta } & \multirow[b]{2}{*}{ Conservación } \\
\hline & 1 & 2 & 3 & 4 & 5 & 6 & \\
\hline 1 & 33 & 25 & 18 & 16 & 6 & 0,5 & Cám. húmed. \\
\hline 2 & 35 & 27 & 21 & 17 & 6,5 & 1,5 & $"$ \\
\hline 4 & 35 & 27 & 21 & 17 & 6,5 & 1,5 & $"$ \\
\hline 4 & 35 & 27 & 21 & 17 & 6,5 & 1,0 & Mojadas \\
\hline 8 & 35 & 27 & 21 & 17 & 6,5 & 2,0 & Cám. húmed. \\
\hline 11 & 35 & 27 & 21 & 17 & 6,5 & 1,0 & $"$ \\
\hline 22 & 35 & 27 & 21 & 17 & 7,0 & 1,0 & Aire \\
\hline 23 & 35 & 27 & 21 & 17 & 7,0 & 1,0 & $"$ \\
\hline 23 & 35,5 & 28 & 21 & 17 & 7,0 & 1,0 & Mojadas \\
\hline
\end{tabular}




\section{Cuadro 4}

\begin{tabular}{|c|c|c|c|}
\hline $\begin{array}{r}\text { Edad } \\
\text { (dias) }\end{array}$ & Probeta A & Probeta B & Conservación \\
\hline 1 & 31 & 19 & Cám. húmed. \\
\hline 2 & 33 & 21 & $"$ \\
\hline 4 & 35 & 22 & $"$ \\
\hline 4 & 35 & 22 & Mojada \\
\hline 11 & 37 & 23 & Cám. húmed. \\
\hline 15 & 37 & 23 & Aire \\
\hline 15 & 37 & 23 & Mojada \\
\hline
\end{tabular}

Se puede comprobar que los valores de reluctancia magnética se estabilizan a partir de las $48 \mathrm{~h}$.

Al comprobar una cierta sistemática en los valores obtenidos, se procedió a calcular en las probetas de mortero la proporción de cemento aluminoso en forma equivalente a $\mathrm{kg} / \mathrm{m}^{3}$ de hormigón y asimismo se efectuó la corrección antes mencionada multiplicando los valores obtenidos con el pachómetro por el factor 1,333 indicado, que tenía en cuenta la mayor superficie de la probeta en relación al palpador.

Los resultados así obtenidos se han representado en la fig. 2, que permite un ajuste suficientemente aceptable y en el que se comprueba que todos los puntos se alinean en función de la proporción en cemento aluminoso.

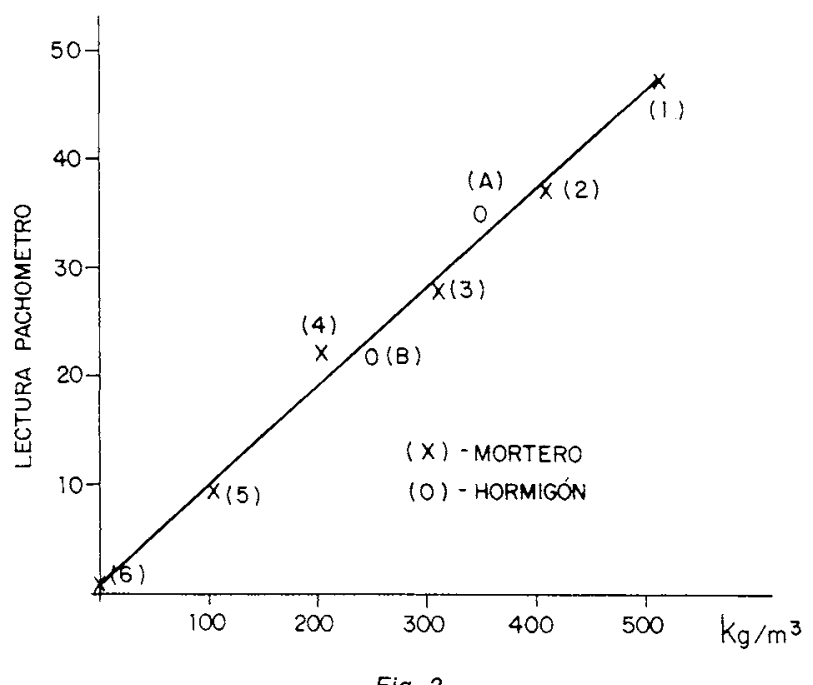

DISCUSIÓN

El hecho de que el cemento aluminoso presente una respuesta a una excitación de tipo magnético, se ha atribuido como debida a los compuestos de hierro que contiene. Estos compuestos son de la familia de la brownmillerrita, ferrito- aluminato tetracálcido $\left[\mathrm{Ca}_{2}\right.$
(Al, $\mathrm{Fe}$ ) $\mathrm{O}_{5}$, compuesto que también forma parte del cemento portland, pero en cantidades menores que en el aluminoso. Además de la brownmillerrita, el cemento aluminoso contiene también óxidos ferrosos y férricos del tipo de la wüstita o de la pleochroita (5).

Asi la graduación que se mide con el pachómetro en función de la proporción de aluminoso añadida, seria debida a la proporción por probeta de todos estos compuestos de hierro al ser el cemento empleado siem. pre el mismo.

Una primera observación optimista de los resultados permite deducir que el pachómetro parece ser no só. lo útil para identificar la presencia de cemento aluminoso, sino incluso parece ser capaz de indicar las porporciones por $\mathrm{m}^{3}$ de hormigón que contiene, al menos en las condiciones controladas de un laboratorio y con el uso de un mismo tipo de cemento. Esta realidad parece sin duda clara, con independencia del contenido en humedad de las probetas.

\section{Viabilidad de aplicación indiscriminada en obra}

Sin embargo, la situación en obra es mucho más compleja, primero porque muchos pachómetros no tienen la posibilidad de una escala cuantitativa como el usado en el presente trabajo, sino que son aparatos de simple SI-NO sin posibilidad de cuantificación. Y en caso de que la tuvieran, siempre habría que proceder a un calibrado previo cuidadoso, del tipo del realizado en el presente trabajo, ya que cada marca comercial puede utilizar rangos de frecuencia de la corriente en alterna diferentes y, por lo tanto, la respuesta al campo magnético puede ser diferente con cada aparato. También porque pueden existir áridos con minerales que presenten una respuesta al campo magnético, similar a la detectada con el aluminoso.

Dado que en los ensayos efectuados sólo se había trabajado con cemento aluminoso sin transformar y con el fin de comprobar si el envejecimiento y carbonatación del cemento aluminoso podria influir en las medidas realizadas, es decir, si seria posible aplicar el método en obras indiscriminadamente, se procedió a medir también en una vigueta, que había sido enviada al Instituto Eduardo Torroja para su análisis. Al medir un trozo de esta vigueta sin armaduras con el pachómetro, se comprobó que la aguja se situaba alrededor del 80 de la escala, es decir, la reluctancia magnética era muy inferior a cualquiera de las probetas preparadas en el laboratorio. Se plantearon pues dos posibles hipótesis, por un lado atribuir a la vigueta cantidades de cemento alrededor a los $1000 \mathrm{~kg} / \mathrm{m}^{3}$, (figura 2) lo que no parecía a simple vista que fuera posible, o bien, que los compuestos a base de hierro se hubieran transformado en otros con mucho más carácter ferromagnético. 
Se procedió entonces a un análisis por rayox $X$ de esta muestra y se comprobó que todo el aluminato cálcico hidratado estaba en su fase cúbica (es decir habia sido transformado a partir de la fase original hexagonal) y también contenía Aragonito, pero no era posible identificar ningún pico correspondiente a la brownmillerita. Es decir, esta sustancia se había transformado en algún compuesto amorfo no identificable por rayos $\mathrm{X}$, que sólo detecta compuestos cristalinos.

La hipótesis que se efectuó entonces es que la brownmillerrita, por el efecto del envejecimiento (humedad y calor), se habría transformado en óxido gamma-férrico $\left(\gamma-\mathrm{Fe}_{2} \mathrm{O}_{3}\right)$ con propiedades magnéticas más acusadas, lo que podría explicar la menor reluctancia magnética detectada. Así pues, parecía deducirse de estos hechos que los hormigones de cemento aluminoso podían aumentar su sensibilidad al campo magnético, al envejecer (humedad y carbonatación) y por tanto al ir transformándose la brownmillerrita en $\gamma \cdot-\mathrm{Fe}_{2} \mathrm{O}_{3}$.

Para comprobarlo se procedió a efectuar un nuevo ensayo que todavía está en curso, y que consistió en coger la probeta de mortero fabricada con el $80 \%$ de cemento aluminoso y $20 \%$ de portland e introducirlo en una estufa a humedad $>90 \%$ y $50{ }^{\circ} \mathrm{C}$ para provocar la transformación de forma acelerada, mientras se media con el pachómetro periódicamente.

Después de 3 meses de ensayo, el pachómetro no detectó ningún cambio, por lo que se procedió a analizarla por rayos $X$, comprobando que sí se habian transformado los aluminatos completamente del hexagonal al cúbico. Así pues, parece que no es sólo necesario que la browmillerrita desaparezca, sino que además puede que resulte imprescindible que se produzca la carbonatación, para que los óxidos de hierro aumenten sus propiedades magnéticas. Para comprobarlo se ha extraido la probeta de la cámara de elevada humedad (que inhibe la carbonatación al mantener los poros del mortero saturados de humedad) y se conserva en estufa a $40^{\circ} \mathrm{C}$. Después de 2 meses en esta situación, el pachómetro parece aumentar muy ligeramente su respuesta que se sitúa alrededor del 40 de la escala. El ensayo se mantiene en curso, mientras se sigue considerando cuál puede ser una explicación lógica a estos comportamientos.

En todo caso la constatación de que una vigueta tomada al azar, y ya envejecida, ha mostrado una respuesta al pachómetro muy superior a la que era de esperar, lleva a concluir por el momento que es mejor conformarse con una simple detección SI-NO sobre la presencia de cemento aluminoso y posponer la posibilidad de además cuantificar la proporción de cemento, para cuando se haya podido realizar una investigación más amplia y sistemática de la limitada aquí realizada, y cuyo objetivo era simplemente encontrar algún soporte científico al uso del pachómetro para (C) Consejo Superior de Investigaciones Científicas

Licencia Creative Commons 3.0 España (by-nc)

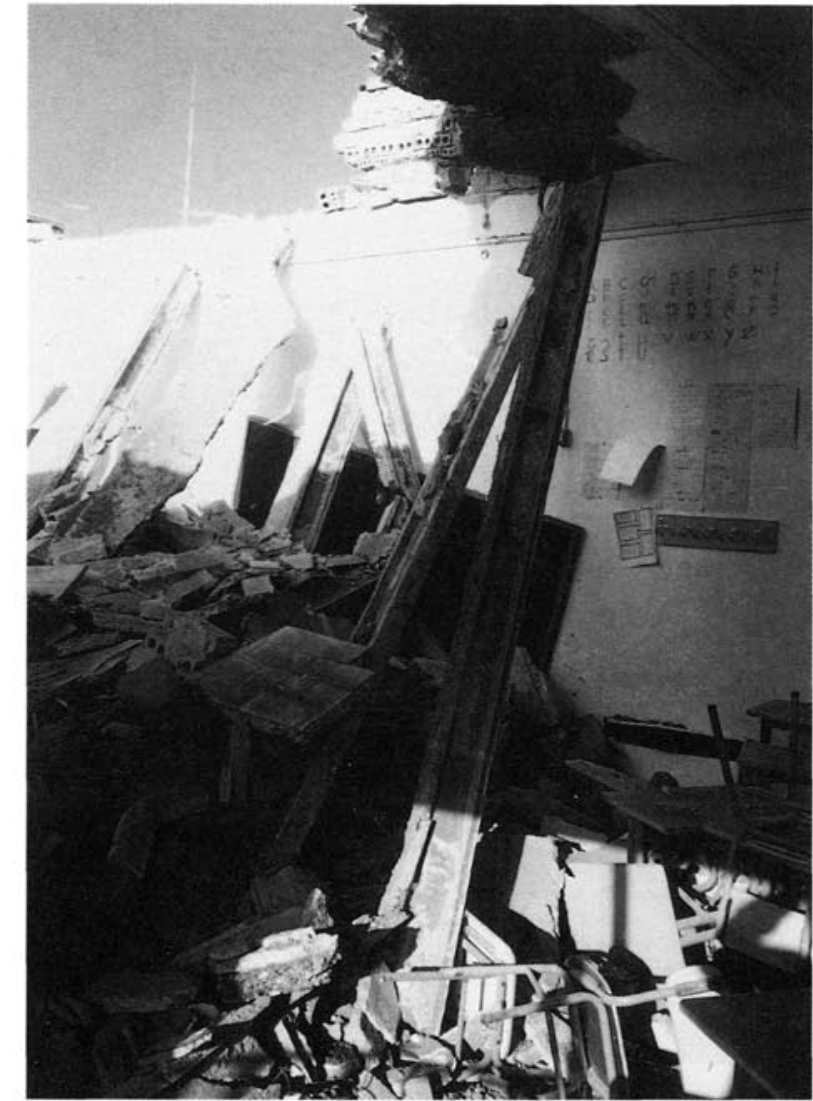

Colapso debido a aluminoso en unas escuelas de Andalucía.

identificar cemento aluminoso en obra. Objetivo que se piensa alcanzado y se espera que pueda contribuir a ayudar a los profesionales del sector.

\section{Otras posibles causas de error}

Ya se ha mencionado la posibilidad de que algunos áridos puedan contener compuestos ferromagnéticos que presentarían también una respuesta positiva al análisis del pachómetro, como sería posiblemente el caso de áridos conteniendo piritas o pirrotinas, de los que se usaron en los años 1970 de la cuenca del Maresme, lo que permitiría por otro lado la identificación de su presencia si la calibración se realiza con un hormigón de cemento portland con áridos no ferromagnéticos.

Además, se ha detectado también que dan una respuesta muy significativa al pachómetro las cenizas volantes (6). Esta respuesta nuevamente se atribuye a que las cenizas contienen magnetita y hematites en distintas proporciones según la procedencia de la ceniza.

Pero por la misma razón se piensa que las escorias de alto horno y algunas puzolanas naturales también pueden contener compuestos ferromagnéticos y por lo tanto ser activas a la señal enviada por el pachómetro. http://informesdelaconstruccion.revistas.csic.es 


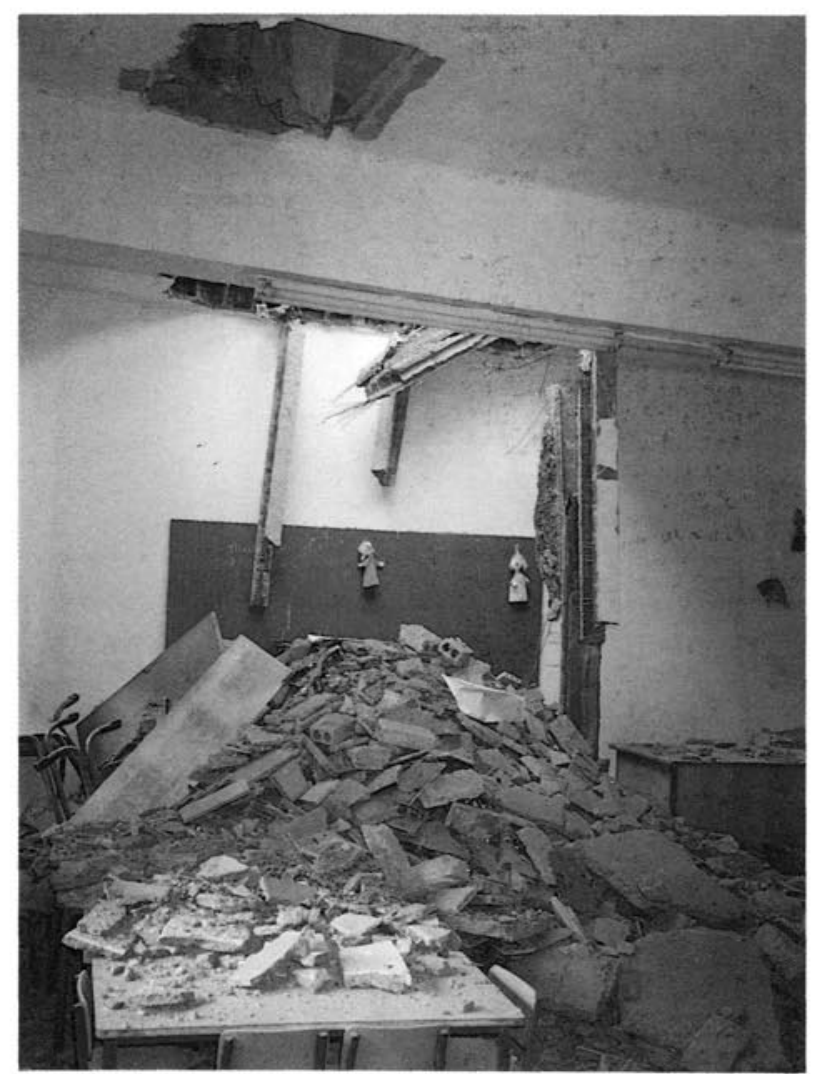

Otro aspecto del colapso de una escuela.

En todo caso es importante resaltar la necesidad de efectuar la calibración del pachómetro con un hormigón fabricado con cemento tipo I, sin ninguna adición y asegurándose también que los áridos no sean reactivos al campo magnético.

El otro aspecto positivo, en cambio, es la posibilidad de identificación que aparece, al menos cualitativa, de identificar la presencia o no de algunas adiciones en los hormigones, mediante el uso de pachómetros adecuados (con escala de espesores de recubrimiento), así como el de identificación de determinados tipos de áridos.

\section{CONCLUSIONES}

A la vista de los hechos presentados puede deducirse que:

1.) Cuando se coloca el palpador de un pachómetro en contacto con hormigón de cemento aluminoso, aparece una respuesta al campo magnético muy superior a la que aparece con cemento portland. Así pues, parece viable poder utilizar este método para identificar la presencia de cemento aluminoso, siempre que se tenga la precaución de no tener cercanas armaduras que puedan interferir en la medida, ni contener el hormigón áridos con propiedades magnéticas, o proporción alguna de adiciones activas, es decir ser de cemento tipo I.
2.) En las condiciones del laboratorio, el método ha resultado además cuantitativo en cuanto a la indicación de la proporción de cemento por $\mathrm{m}^{3}$ de hormigón. En cambio los resultados en una vigueta envejecida no han permitido esta cuantificación, no habiéndose encontrado todavia una explicación suficiente para ello.

3.) Debido a lo limitado de la investigación, la respuesta con otros aparatos y otras condiciones puede resultar diferente de la aqui presentada, por lo que es necesario calibrar cualquier aparato que se vaya a utilizar para este propósito y así verificar que es sensible a la presencia de cemento aluminoso.

\section{AGRADECIMIENTOS}

Los autores agradecen a D. Anselmo Garay, del Instituto Eduardo Torroja, todos sus comentarios sobre el uso y limitaciones de los pachómetros y a los Dres. Sara Goñi y José Luis Sagrera, también pertenecientes al Instituto, por sus interpretaciones de los análisis de infrarrojos y rayos $X$.

\section{BIBLIOGRAFIA}

(1) C. ANDRADE, S. GOÑI, J. L. SAGRERA y C. ALONSO - Procesos de degradación del cemento aluminoso fundido - Revista BIA n. 143 - Marzo 1991. pag. 83.

(2) T. VAZQUEZ, F. TRIVIÑO, A. RUIZ DE GAUNA - Estudio de las transformaciones del cemento aluminoso hidratado, influencia del anhídrido carbónico, temperatura, humedad y adición de caliza en polvo - Monografia n. 334 del ICCET (1976).

(3) ITEC - Recomendaciones para el reconocimiento sistemático y el diagnóstico rápido de viguetas construidas con cemento aluminoso - Barcelona 1991.

(4) A. GARAY - Métodos no destructivos de estudio del hormigón - Cursos CEMCO - ICCET.

(5) M. PEREZ - Aspectos físico-químicos y estabilización de los aluminatos cálcicos resultantes de la hidratación del cemento aluminoso - Tesis Doctoral - Univ. Complutense de Madrid (1981).

(6) CH. W. FARRELL, K.C. HOVER, P.W. PLUMLEY Natural remanent magnetization of portland cement concrete - Cement and Concrete Research, vol. 21 pp. 489-495 (1991). 